\title{
The Influence of Harsh Parenting on Middle School Students' Learning Engagement: The Mediating Effect of Perceived Self-Efficacy in Managing Negative Affect and the Moderating Effect of Mindfulness
}

\author{
Jiaxin Zhang, Pengfei Yue* \\ School of Educational Science, Xinyang Normal University, Xinyang, China \\ Email: *ypf07@126.com
}

How to cite this paper: Zhang, J. X., \& Yue, P. F. (2021). The Influence of Harsh Parenting on Middle School Students' Learning Engagement: The Mediating Effect of Perceived Self-Efficacy in Managing Negative Affect and the Moderating Effect of Mindfulness. Psychology, 12, 1473-1489. https://doi.org/10.4236/psych.2021.1210093

Received: September 7, 2021

Accepted: October 11, 2021

Published: October 14, 2021

Copyright $\odot 2021$ by author(s) and Scientific Research Publishing Inc. This work is licensed under the Creative Commons Attribution International License (CC BY 4.0).

http://creativecommons.org/licenses/by/4.0/

\begin{abstract}
Objectives: In previous studies, there was still a lack of discussion on the impact mechanism of harsh parenting on learning engagement. This study discussed the mediating role of perceived self-efficacy in managing negative affect (NEG) between harsh parenting and learning engagement according to the Development-in-Sociocultural-Context Model for Children's Engagement in Learning, and investigated the moderating role of mindfulness between them. Methods: A total of 742 junior middle school students were tested by Harsh Parenting Questionnaire, The Scale of Regulatory Emotional Self-efficacy, Utrecht Work Engagement Scale-student and Mindfulness Attention Awareness Scale. Results: 1) Harsh parenting negatively predicts learning engagement; 2) NEG plays a mediating role between harsh parenting and learning engagement; 3) Mindfulness can moderate the relationship between harsh parenting and learning engagement. Specifically, compared with low mindfulness individuals, harsh parenting has a stronger negative predictive effect on learning engagement of high mindfulness individuals. Mindfulness is a vulnerable factor of stress in the influence of harsh parenting on learning engagement. Conclusions: Harsh parenting can reduce individual learning engagement by damaging NEG, and the protective effect of mindfulness on learning engagement of harsh parented individuals is limited.
\end{abstract}

\section{Keywords}

Harsh Parenting, Perceived Self-Efficacy in Managing Negative Affect, 
Learning Engagement, Mindfulness, Stress-Vulnerability Hypothesis

\section{Introduction}

Learning engagement refers to the full, stable and continuous positive state of individuals in learning (Schaufeli et al., 2002). It can predict not only the current academic performance of an individual (Wei et al., 2014), but also longitudinally his or her enrollment in further studies and/or work situation ten years later (Alexander et al., 1997), rendering a far-reaching impact on individual development. Previous studies have shown that environmental factors (such as teacher support and rearing style) (Jia et al., 2020; Li, 2018) and individual factors (such as gratitude) (Wen et al., 2010) will affect individual learning engagement. However, how harsh parenting with extreme lack of love and warmth affects learning engagement has not been fully discussed. Previous studies have found that there is a negative relationship between strict parenting and learning engagement (Zhou et al., 2014; Li, 2012). Since harsh parenting and strict parenting are two overlapping concepts (Wang et al., 2016) with a shared element of authoritarian parenting (such as control with force over children, guidance with lack of reasoning, hostile language, etc.), harsh parenting is likely to have a negative relationship with learning engagement, too. Later, Zhang (2020) confirms that there is a negative relationship between harsh parenting and learning engagement, but the discussion on the mechanism is not sufficient. According to the Developmentin-Sociocultural-Context Model for Children's Engagement in Learning (Wang et al., 2019), Harsh parenting not only directly affects learning engagement, but also indirectly affects learning engagement by affecting perceived self-efficacy in managing negative affect (NEG). This study intends to test this indirect path and examine the role of mindfulness. It is expected to enrich the exploration of the mechanism of harsh parenting affecting learning engagement, and provide a theoretical basis for the protection and cultivation of middle school students' learning engagement.

\subsection{The Relationship between Harsh Parenting and Learning Engagement}

The Development-in-Sociocultural-Context Model for Children's Engagement in Learning assumes that parental rearing can directly affect learning engagement (Wang et al., 2019). According to the model, harsh parenting, a negative parenting style, is likely to have an adverse impact on learning engagement. The relationship between harsh parenting and learning engagement can be explained by Self-Determination Theory. Self-Determination Theory assumes that people are positive organisms and naturally have rich intrinsic motivation tendencies, which can enable people to actively integrate themselves, improve themselves and continue to learn. However, maintaining and enhancing this intrinsic ten- 
dency requires supportive conditions, and this tendency is very easy to be destroyed by various non supportive conditions (Ryan \& Deci, 2017). Research shows that supportive conditions such as providing choices and acknowledging feelings that enable people to have a greater sense of autonomy can enhance intrinsic motivation, while non-supportive conditions such as threats, instructions and imposed goals can weaken intrinsic motivation (Deci \& Ryan, 1985). Harsh parenting is a kind of non-supportive condition existing in the family. It refers to parents' rude behavior, emotion and attitude towards children, including not only explicit verbal and physical attacks such as yelling and beating, but also psychological attacks such as refusing and denying children's psychological needs, as well as violations of children's rights, punishment of children with no justification, and other forced/controlled behaviors (Wang et al., 2016). Under such environmental conditions, parents control children in a rough way, with children as individuals forced to carry out learning activities, their psychological needs not met, and their intrinsic motivation for learning seriously damaged, thus the level of learning engagement is often low. In addition, Grolnic et al. (1997) also finds that children of controller-type parents have lower intrinsic motivation than those of parents supporting children's autonomy. In conclusion, because harsh parenting will reduce the intrinsic motivation of individual learning, the level of learning engagement of individuals who are harshly parented is often low. Empirical research also shows that there is a negative relationship between harsh parenting and learning engagement (Zhang, 2020). Therefore, this study puts forward hypothesis 1: Harsh parenting can negatively predict learning engagement.

\subsection{The Mediating Role of NEG}

The Development-in-Sociocultural-Context Model for Children's Engagement in Learning also assumes that parental rearing can not only directly affect learning engagement, but also indirectly affect it by affecting children's individual emotional regulation (Wang et al., 2019). According to the model, harsh parenting is likely to indirectly affect learning engagement by affecting individual NEG.

Firstly, harsh parenting negatively predicts NEG. NEG refers to the degree of self-confidence that individuals can effectively adjust their negative emotions in the face of negative emotions (Caprara et al., 2008). It is an individual's sense of efficacy in the field of emotion regulation. Bandura et al. (1997) assumes that active mastery experience is the most important source of individual self-efficacy. Successful experience can improve individual self-efficacy. On the contrary, failed experience will reduce individual self-efficacy. Previous studies have shown that parents' harsh treatment of children, such as punishment, lie, abuse and control, will damage children's emotional regulation ability, and children are prone to negative emotional experiences such as anxiety and depression (Callahan et al., 2011; Brody et al., 2014; Liu \& Wei, 2020; Wei \& Liu, 2021). When the individual's emotion regulation ability is not enough to effectively regulate these negative emotions, the failed emotion regulation experience will reduce the individual NEG. 
Therefore, harsh parenting negatively predicts NEG.

Secondly, NEG positively predicts learning engagement. Short-Term Mood Regulation Theory holds that when individuals experience negative emotions in the process of pursuing goals (such as learning tasks), for individuals, repairing current emotions is prior to pursuing long-term goals. In order to repair current negative emotions, individuals give up behaviors with positive long-term results (such as learning) (Sirois \& Pychyl, 2013). High NEG individuals can better regulate negative emotions, so they are likely to be able to better participate in learning activities. Relevant studies also show that individuals with weak emotion regulation ability are prone to negative emotions such as boredom and shame during learning (Gong et al., 2016), which has an adverse impact on learning engagement. For example, shame can easily lead students to lose interest in learning (Xiong et al., 2011); Boredom will damage students' learning motivation and hinder individuals from using flexible and positive learning strategies (Lewis et al., 2009). In conclusion, NEG positively predicts learning engagement.

Therefore, this study puts forward hypothesis 2 : NEG plays a mediating role between harsh parenting and learning engagement.

\subsection{The Moderating Role of Mindfulness}

According to the Organismic Integration Theory (Ryan \& Deci, 2017), human motivation is a continuum of autonomous-controlled motivations. Different positions on this continuum represent the degree of relative autonomy experienced by individuals in a certain behavior situation. For example, the motivation form at the rightmost end of the continuum is intrinsic regulation, which is the motivation with the highest degree of autonomy, that is, intrinsic motivation. Although the support from their social backgrounds is very important for individuals to maintain intrinsic motivation, people can still adjust themselves through personal internal processes (such as mindfulness) to realize the transformation from extrinsic motivation to intrinsic motivation when the external situation is unfavorable (Ryan et al., 2012). According to the Organismic Integration Theory, when an individual is under the condition of harsh parenting, it is easy to form the motivation of external regulation, and mindfulness can promote the purposeful transformation of controlled motivation to autonomous motivation (Deci \& Ryan, 2008; Ryan \& Deci, 2017). Therefore, among the individuals who are brutally raised, the high mindfulness people may have stronger autonomy of learning motivation and higher level of learning engagement. However, can mindfulness still play a role under the pressure of highly harsh parenting? Stress-Buffering Hypothesis and Stress-Vulnerability Hypothesis give different predictions. The Stress-Buffering Hypothesis holds that individuals' positive factors will buffer the impact of stress, protect individuals to better cope with stress, and individuals with positive qualities adapt well in both low and high stress environments (Laborde et al., 2011; Martins et al., 2010). According to the Stress-Buffering Hypothesis, it can be inferred that high mindfulness individuals are less 
affected by harsh parenting, and always maintain a high level of learning engagement in the process from low harsh parenting to high harsh parenting, while learning engagement of low mindfulness individuals declines rapidly when they encounter high harsh parenting. With the increase of harsh parenting intensity, the gap between high mindfulness individuals and low mindfulness individuals in learning engagement level gradually widens. However, the StressVulnerability Hypothesis holds that positive factors often lose their buffering effect in high-pressure environment. Although individuals with positive quality adapt well in low-pressure environment, their adaptation level deteriorates rapidly in high-pressure environment (Li et al., 2012). According to the StressVulnerability Hypothesis, it can be inferred that under low harsh parenting, high mindfulness individuals have a higher level of learning engagement than low mindfulness individuals, but under high harsh parenting, regardless of mindfulness, the level of learning engagement becomes lower. With the increase of harsh parenting intensity, the gap of learning engagement between high mindfulness individuals and low mindfulness individuals gradually narrowed.

Accordingly, this study puts forward hypothesis 3: Mindfulness can moderate the relationship between harsh parenting and learning engagement. In view of the relative lack of previous studies on the interaction between harsh parenting and mindfulness, this study only makes exploratory analysis on the specific mode of moderation ("Stress-Buffering vs. Stress-Vulnerability") without making specific assumptions (see Figure 1 for the schematic diagram of the two modes).

In conclusion, this study aims to explore the mediating (NEG) and moderating (mindfulness) mechanisms of harsh parenting on learning engagement (Figure 2) (Harsh parenting affects learning engagement through the mediating effect of NEG, while mindfulness moderates the direct effect of harsh parenting on learning engagement). In order to better answer the question of "how" harsh parenting affects learning engagement and "when" this influence is stronger or weaker, and provide a theoretical basis for the protection and cultivation of middle school students' learning engagement.

\section{Methods}

\subsection{Participants}

We recruited 800 students from three junior middle schools in Henan Province, China. Then, we sort out the invalid questionnaires in turned questionnaires: removing 22 questionnaires in which the number of missed questions exceeds two thirds of the total questionnaire and 36 questionnaires with the same option selected in the entire questionnaire, and finally we get 742 valid questionnaires. The calculated recovery rate is $92.75 \%$. The average age of all participants was 13.13 years $(\mathrm{SD}=1.03)$, including 349 boys $(47.0 \%)$ and 393 girls $(53.0 \%) ; 361$ $(48.7 \%)$ in grade one, $178(24.0 \%)$ in grade two and $203(27.4 \%)$ in grade three; 47 only child (6.3\%) and 695 nononly child (93.7\%); 624 rural students (84.1\%) 


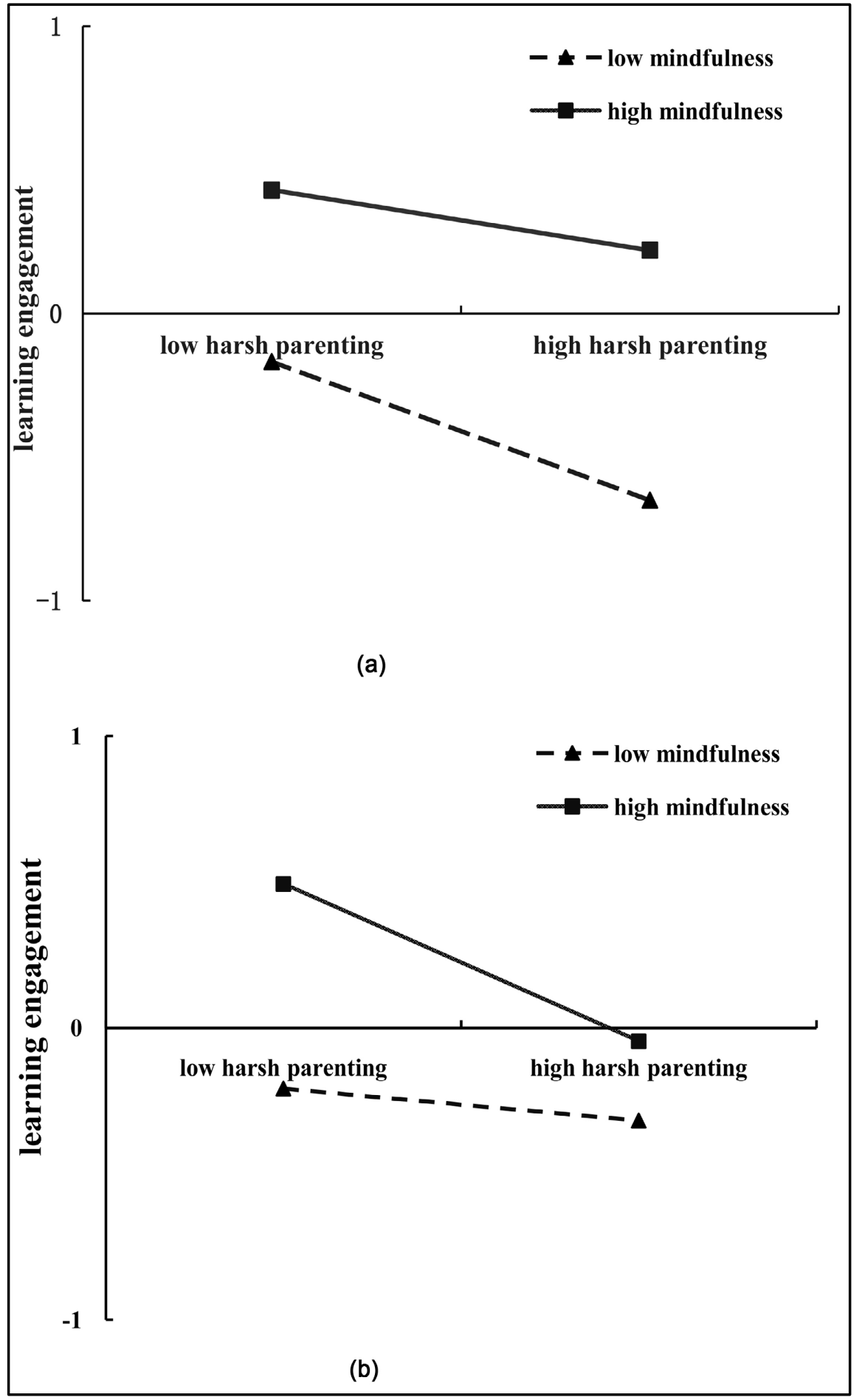

Figure 1. Schematic diagram of two moderation modes. (a) The mode of "stress-buffering"; (b) The mode of "stress vulnerability".

and 118 urban students (15.9\%). In addition, $44.3 \%$ of the parents' annual income was less than 30,000 yuan, $36.3 \%$ of the parents' annual income was between 30,000 yuan and 80,000 yuan, and only $19.4 \%$ of the parents' annual income exceeded 80,000 yuan. 


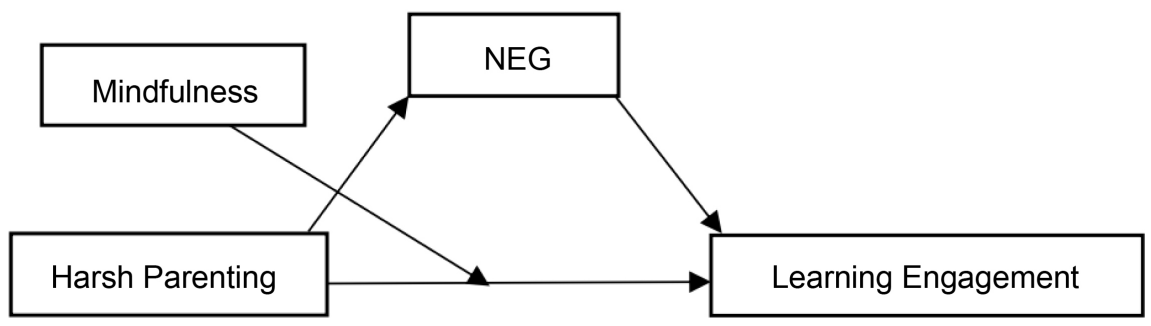

Figure 2. Hypothetical model of the influence of harsh parenting on learning engagement.

\subsection{Measures}

\subsubsection{Harsh Parenting}

Harsh parenting was assessed by a Harsh Parenting Questionnaire with four items (such as "when I did something wrong or made my parents angry, he (or she) would lose temper or even yell at me") (Wang et al., 2017). Children rated the four items for each parent's behaviors. Participants were asked to rate how much they agreed with each of the following statements on a 5-point scale ranging from 1 = "Strongly disagree" to 5 = "Strongly agree". The higher score indicates that the higher the degree of harsh parenting of individuals by their parents. In this study, the Cronbach's a coefficient of the scale was 0.78 .

\subsubsection{Perceived Self-Efficacy in Managing Negative Affect (NEG)}

NEG was assessed by subscale of The Scale of Regulatory Emotional Self-efficacy with eleven items (such as "I can control my negative emotions when scolded by my parents or other important people") that were compiled by Caprara et al. (2008) and revised by Wang et al. (2013). Children rated the eleven items according to their self-confidence in regulating negative emotions. Participants were asked to rate how much they agreed with each of the following statements on a 5 -point scale ranging from $1=$ "Strongly disagree" to $5=$ "Strongly agree". The higher score indicates that the higher the level of individual regulating negative emotions. In this study, the Cronbach's a coefficient of the subscale was 0.91 .

\subsubsection{Learning Engagement}

Learning engagement was assessed by The Utrecht Work Engagement Scalestudent with seventeen items (such as "I am full of enthusiasm for learning") that were compiled by Schaufeli et al. (2002) and revised by Fang et al. (2008). Children rated the seventeen items according to their level of learning engagement. Participants were asked to rate how much they agreed with each of the following statements on a 7-point scale ranging from $1=$ "Strongly disagree" to 7 = "Strongly agree". The higher score indicates that the higher the level of individual learning engagement. In this study, the Cronbach's a coefficient of the scale was 0.96 .

\subsubsection{Mindfulness}

Mindfulness was assessed by Mindfulness Attention Awareness Scale with fifteen items (such as "I may not be aware of certain emotions until they last for some 
time") that were compiled by Brown and Ryan (2003) and revised by Chen et al. (2012). The children rated the fifteen items according to their level of mindfulness. Participants were asked to rate how much they agreed with each of the following statements on a 6 -point scale ranging from $1=$ "Strongly agree" to $6=$ "Strongly disagree". The higher score indicates that the higher the level of individual mindfulness. In this study, the Cronbach's a coefficient of the scale was 0.88 .

\subsection{Procedures}

The experimenter of this study was conducted by trained graduate students majoring in psychology. The informed consent of school leaders, teachers and students was obtained before the test. The cluster random sampling method was used to carry out the group test with the class as the unit, and the anonymous filling method was adopted. The participants were required to answer truthfully and independently. After the test, the questionnaire was collected on the spot. It takes about 20 minutes for the subjects to complete the whole questionnaire. The collected data were statistically analyzed by spss26.0. Descriptive statistics mean difference tests and correlations analysis were first calculated. Then the SPSS PROCESS macro was used to test our model, include how (mediating effect) and when (moderating effect) of harsh parenting influences learning engagement. do not revise any of the current designations.

\section{Results}

\subsection{Common Method Bias Test}

In this study, Harman single-factor method was used to analyze the data. The results show that there were 6 common factors being extracted and the first common factor explained $29.37 \%$ of the total variance, it was far less than $40 \%$ (Podsakoff et al., 2003). It can be inferred that common method bias did not have much impact on the results of this study.

\subsection{Correlation Description}

Descriptive statistics and correlation analysis were performed on each variable in the study (Table 1). Harsh parenting is negatively correlated with learning engagement, so hypothesis 1 of this study is verified; harsh parenting was also negatively correlated with NEG and mindfulness; NEG was positively correlated with learning engagement and mindfulness; there is a positive correlation between learning engagement and mindfulness. In addition, gender was significantly correlated with NEG; age was significantly correlated with mindfulness; grade was significantly correlated with harsh parenting, NEG as well as mindfulness; whether the only child was significantly correlated with harsh parenting; family location was significantly correlated with learning engagement. Therefore, in the follow-up analysis, gender, age, grade, whether the only child and family location are treated as control variables. 
Table 1. Descriptive statistics and intercorrelations between all study variables.

\begin{tabular}{lccccc}
\hline \multicolumn{1}{c}{ Variables } & $\mathrm{M} \pm \mathrm{SD}$ & 1 & 2 & 3 & 4 \\
\hline 1) harsh parenting & $1.76 \pm 0.78$ & 1 & & & \\
2) NEG & $3.11 \pm 0.90$ & $-0.18^{* *}$ & 1 & & \\
3) learning engagement & $3.78 \pm 1.39$ & $-0.20^{* *}$ & $0.46^{* *}$ & 1 & \\
4) mindfulness & $3.67 \pm 1.00$ & $-0.24^{* *}$ & $0.32^{* *}$ & $0.29^{* *}$ & 1 \\
\hline
\end{tabular}

Note: $\mathrm{N}$ for all $=742 ; \mathrm{M}=$ mean. $\mathrm{SD}=$ standard deviations; ${ }^{*} p<0.05,{ }^{* *} p<0.01$, the same below.

\subsection{The Mediating Effect Test of NEG}

In this study, model 4 in the PROCESS macro developed by Hayes (2013) was used to analyze the mediating effect with harsh parenting as the independent variable, NEG as the mediating variable and learning engagement as the dependent variable under the control of gender, age, grade, whether the only child and family location. The results show that (Table 2): harsh parenting negatively predicts learning engagement $(\beta=-0.12, p<0.01)$; harsh parenting negatively predicts NEG $(\beta=-0.17, p<0.01)$, NEG positively predicts learning engagement $(\beta=$ $0.43, p<0.01)$. The significance test of mediating effect shows that $\mathrm{a} \times \mathrm{b}=-0.07$, boot $\mathrm{SE}=0.02,95 \%$ confidence interval is $[-0.11,-0.04]$, excluding 0 . This result shows that NEG plays a significant mediating role between harsh parenting and learning engagement. The hypothesis 2 of this study has been verified. The mediating effect $(-0.07)$ accounted for $37.90 \%$ of the total effect $(-0.19)$ (Table 3$)$.

\subsection{The Moderating Effect Test of Mindfulness}

In this study, model 5 (assuming that the direct path is moderated) in the PROCESS macro is used to analyze the moderating effect. Under the control of gender, age, grade, whether the only child and family location, harsh parenting is the independent variable, NEG is the mediating variable, learning engagement is the dependent variable and mindfulness is the moderating variable. The results show that (Table 4): harsh parenting negatively predicts learning engagement $(\beta=-0.12, p<0.01)$, mindfulness positively predicts learning engagement $(\beta=$ $0.13, p<0.01$ ), and the interaction of harsh parenting and mindfulness has a significant predictive effect on learning engagement $(\beta=-0.10, p<0.01), 95 \%$ confidence interval is $[-0.16,-0.04]$, excluding 0 . This result shows that mindfulness can moderate the relationship between harsh parenting and learning engagement. The hypothesis 3 of this study is verified.

In order to reveal how mindfulness moderates the impact of harsh parenting on learning engagement, high and low groups $( \pm 1 \mathrm{SD})$ are divided according to the quantity of mindfulness, a simple slope test is carried out, and a simple effect analysis diagram is drawn (Figure 3). The results showed that when the individual mindfulness level is low $(-1 \mathrm{SD})$, the predictive effect of harsh parenting on learning engagement is not significant $\left(b_{\text {simple }}=-0.05, p=0.28\right)$, and the $95 \%$ confidence interval is $[-0.13,0.04]$; When the individual mindfulness level is high (+1SD), harsh parenting has a strong negatively predictive effect on learning engagement $\left(b_{\text {simple }}=-0.27, p<0.01\right)$, and the $95 \%$ confidence interval is 
Table 2. The mediating effect test of NEG.

\begin{tabular}{ccccccc}
\hline \multirow{2}{*}{ Variables } & \multicolumn{3}{c}{ NEG } & \multicolumn{3}{c}{ Learning Engagement } \\
\cline { 2 - 7 } & $\beta$ & SE & $\mathrm{t}$ & $\beta$ & $\mathrm{SE}$ & $\mathrm{t}$ \\
\hline harsh parenting & -0.17 & 0.04 & $-4.59^{* *}$ & -0.12 & 0.03 & $-3.54^{* *}$ \\
NEG & & & & 0.43 & 0.03 & $12.93^{* *}$ \\
$\mathrm{R}^{2}$ & & & & & 0.24 & \\
$\mathrm{~F}$ & & 0.06 & & & $32.42^{* *}$ & \\
\hline
\end{tabular}

Note: All variables in the model have been standardized, the same below.

Table 3. Mediating effect test result.

\begin{tabular}{cccc}
\hline Path & Effect Size & $\begin{array}{c}\text { Relative } \\
\text { Effect Quantity }\end{array}$ & $\begin{array}{c}\text { Bootstrap } \\
(95 \% \text { CI })\end{array}$ \\
\hline indirect path & -0.07 & $37.90 \%$ & {$[-0.11,-0.04]$} \\
direct path & -0.12 & $62.10 \%$ & {$[-0.18,-0.05]$} \\
\hline
\end{tabular}

Table 4. The moderating effect test of mindfulness.

\begin{tabular}{cccc}
\hline Variables & \multicolumn{3}{c}{ Learning Engagement } \\
\cline { 2 - 4 } & $\beta$ & $\mathrm{SE}$ & $\mathrm{t}$ \\
\hline harsh parenting & -0.12 & 0.03 & $-3.55^{\star *}$ \\
mindfulness & 0.13 & 0.03 & $3.86^{* *}$ \\
harsh parenting $\times$ mindfulness & -0.10 & 0.03 & $-3.30^{* *}$ \\
$\mathrm{R}^{2}$ & & 0.26 & \\
$\mathrm{~F}$ & & $29.12^{* *}$ & \\
\hline
\end{tabular}

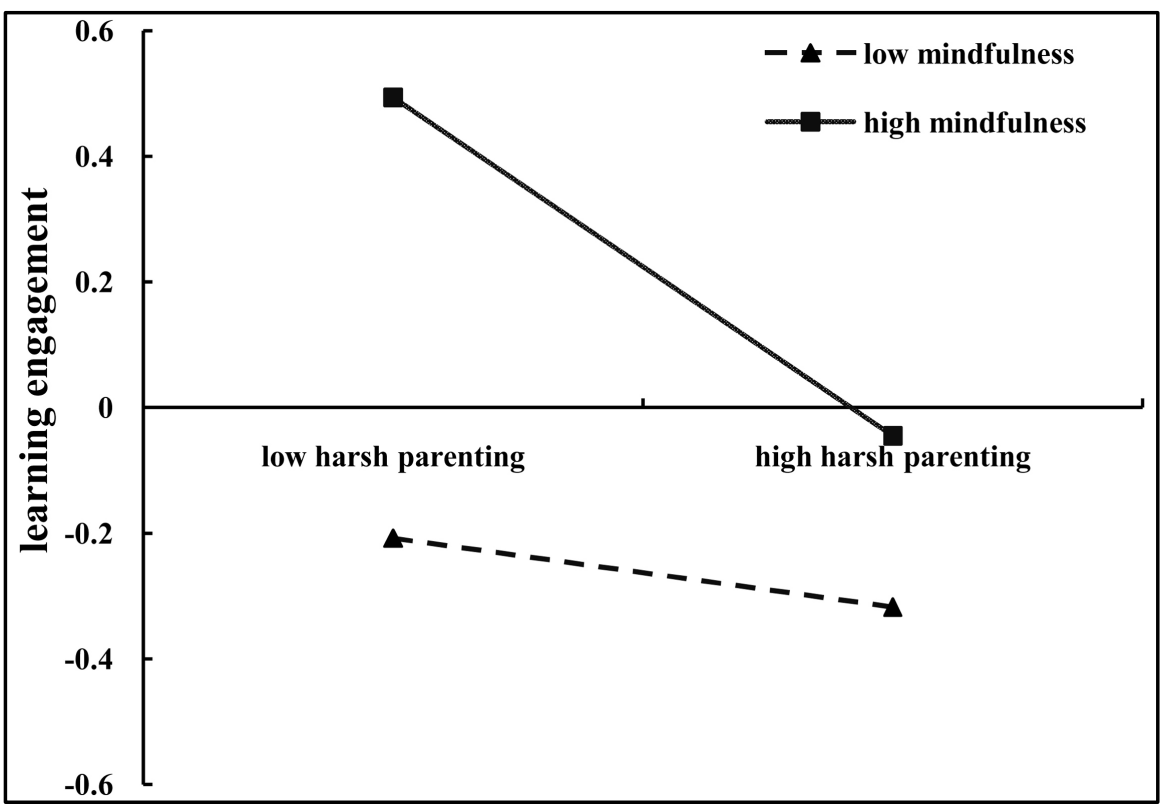

Figure 3. Mindfulness as a moderator in the relationship between harsh parenting and learning engagement. 
$[-0.38,-0.17]$. This result supports the prediction of the "Stress-Vulnerability Hypothesis", which shows that mindfulness is a stress vulnerability factor in the impact of harsh parenting on learning engagement.

\section{Discussion}

\subsection{The Direct Effect of Harsh Parenting on Learning Engagement}

This study finds that harsh parenting negatively predicts learning engagement. This result is consistent with previous studies (Zhang, 2020), and also supports the prediction of Self-Determination Theory (Ryan \& Deci, 2017). The individuals with harsh parenting tend to form controlled motivation, so the level of learning engagement is generally low. There may be various reasons for the low level of learning engagement of harshly raised individuals. According to Attachment Theory, unsafe parent-child attachment leads to children's negative internal representation of themselves, others and interpersonal communication, and then damages the interpersonal function outside the family through this negative internal work model (Bretherton \& Munholland, 2008). Harsh parenting will break the external parent-child relationship, which may cause children to form negative internal representations of themselves and their parents and damage important interpersonal relationships in the learning situation (such as teacher-student relationship and classmate communication), and then cause a low level of learning engagement. Harsh parenting can also damage the individual's effort-control ability (Wang et al., 2020). Individuals with strong effort-control ability are more able to inhibit bad behaviors in the learning situation (such as children ruminate the scenario when they are attacked by their parents' body or speech), activate behaviors consistent with the learning situation, and better focus on learning activities (Kochanska et al., 2000). Because harsh parenting impairs this important ability, it will reduce the level of individual learning engagement.

\subsection{The Mediating Role of NEG}

This study finds that NEG plays a mediating role between harsh parenting and learning engagement, and the mediating effect accounts for $37.90 \%$ of the total effect, indicating that NEG can explain a considerable part of the variation between harsh parenting and learning engagement. The establishment of this mediating effect supports the Development-in-Sociocultural-Context Model for Children's Engagement in Learning (Wang et al., 2019). Harsh parenting will not only directly affect learning engagement, but also indirectly affect learning engagement by affecting NEG. This result also answers the question of "how" harsh parenting affects learning engagement. On the one hand, harsh parenting will reduce individual NEG. Harsh parenting reduces the ability of individual emotion regulation, and failed emotion regulation experience will lead to lower NEG. In addition, some studies have found that parents who adopt harsh parenting often have the characteristics of emotional instability (Clark et al., 2000) 
and difficulty in emotional regulation (Deater-Deckard et al., 2010), so it is difficult for these parents to demonstrate reasonable emotional regulation methods to children. From the perspective of Social Learning Theory (Bandura, 1976), individuals can not learn effective emotion regulation methods from their parents, so their own emotion regulation ability is often low, and then the failed emotion regulation experience will reduce the individual NEG. On the other hand, high NEG students can adjust their negative emotions well and are less disturbed by negative emotions, so they can better devote themselves to learning. Zhang (2020) finds that harsh parenting can affect learning engagement by affecting individual intentional self-adjusting and perceived teacher support. This study finds that NEG is also an important mediating mechanism of harsh parenting affecting learning engagement. It gives a possible explanation for the relationship between them from the perspective of emotional regulation, which enriches the exploration of the mediating mechanism of harsh parenting affecting learning engagement. In future research, we can continue to test whether other factors (such as cognitive skills and social skills) can also mediate the relationship between harsh parenting and learning engagement according to the Development-in-Sociocultural-Context Model for Children's Engagement in Learning (Wang et al., 2019).

\subsection{The Moderating Role of Mindfulness}

This study finds that among the individuals who were harshly raised, those with high mindfulness had a higher level of learning engagement than those with low mindfulness. This result supports the prediction of Organismic Integration Theory (Deci \& Ryan, 2008; Ryan \& Deci, 2017), which shows that mindfulness can transform the controlled learning motivation formed by harsh parenting into more autonomous learning motivation, and improve the level of learning engagement. Siff (2014) assumes that autonomy is a function of integration. In order to achieve integration, people need to be able to freely deal with specific actions and find reasons for recognition. Mindfulness is related to people's ability to pay attention to the current internal and external experience. It enables people to obtain greater insight and self-reflection, and can make individual perception and values more consistent with their own behavior, so their behavior is more autonomous. In addition, this study also finds that with the improvement of mindfulness level, the predictive effect of harsh parenting on learning engagement increases from insignificant $\left(b_{\text {simple }}=-0.05, p=0.28\right)$ to significantly negative $\left(b_{\text {simple }}=-0.27, p<0.01\right)$. This result answers the question of "when" the impact of harsh parenting on learning engagement is stronger or weaker. At the same time, this result supports the prediction of the Stress-Vulnerability Hypothesis (Li et al., 2012), showing that mindfulness, a positive factor, has lost its buffering effect in the stress situation of harsh parenting. Although high mindfulness has a high level of learning engagement under low harsh parenting, the level of learning engagement under high harsh parenting decreases 
rapidly. With the increase of harsh parenting intensity, the gap between high mindfulness and low mindfulness in learning engagement gradually narrows. This suggests that mindfulness is a vulnerable factor of stress in the impact of harsh parenting on learning engagement. Therefore, we should not overestimate the protective effect of mindfulness on the learning engagement of harshly raised individuals. Future research can continue to explore whether there are other more effective protective factors to protect the learning engagement of harshly raised individuals.

\subsection{Educational Suggestions}

This study is of great significance to the protection and cultivation of middle school students' learning engagement. Firstly, according to the direct effect in this study, harsh parenting negatively predicts learning engagement. It shows that parents' educational concept of "no beating, no talent" and harsh educational methods can not effectively improve children's learning engagement. On the contrary, they will reduce children's learning engagement. Therefore, in order to protect children's learning engagement, parents should learn scientific educational ideas with an open mind and reduce harsh parenting. Secondly, according to the mediating effect, harsh parenting affects learning engagement by reducing children's NEG. Parents can discuss on more methods and experiences of emotion regulation with their children, because good emotion regulation helps to improve learning engagement. Finally, according to the moderating effect, mindfulness is a vulnerable factor of stress in the impact of harsh parenting on learning engagement. This shows that the protective effect of mindfulness on learning engagement of harshly parented individuals is limited, and mindfulness will lose its effect in the situation of high harsh parenting. This also reflects that harsh parenting has a strong impact on children's learning engagement. Mindfulness alone is difficult to offset the negative effects of harsh parenting. Therefore, parents need to be aware of their major responsibilities in parenting behavior and reduce harsh parenting.

\section{Limitation}

Although results are promising, several limitations should be addressed in the present study. Firstly, although this study has a relatively solid theoretical basis for the investigation of relevant variables, it still belongs to cross-sectional study. Whether the research results are reliable needs to be further investigated by the research design with causal inference such as longitudinal study. Secondly, harsh parenting in this study does not distinguish between father's harsh parenting and mother's harsh parenting, but the effects of father's harsh parenting and mother's harsh parenting on middle school students' learning engagement may be different. Future research can distinguish father's harsh parenting from mother's harsh parenting, and deeply explore the similarities and differences of the effects of these two kinds of harsh parenting on individual learning engagement. Thirdly, 
harsh parenting was measured just in children's perceptions. Future research should integrate multiple information sources (such as children and parents) to collect data, so as to better measure relevant variables.

\section{Conclusion}

1) Harsh parenting negatively predicts learning engagement;

2) NEG plays a mediating role between harsh parenting and learning engagement;

3) Mindfulness can moderate the relationship between harsh parenting and learning engagement. Specifically, compared with low mindfulness individuals, harsh parenting has a stronger negative predictive effect on learning engagement of high mindfulness individuals. Mindfulness is a vulnerable factor of stress in the influence of harsh parenting on learning engagement.

\section{Acknowledgements}

The authors would like to thank the reviewers for their valuable comments on the manuscript.

\section{Funding}

This study was supported by Innovation Team Support Program of Philosophy and Social Science in Colleges and Universities of Henan Province (2018-CXTD04) and the Key Project of Educational Science Planning in Henan Province (2021JKZD11).

\section{Conflicts of Interest}

The authors declare no conflicts of interest regarding the publication of this paper.

\section{References}

Alexander, K. L., Entwisle, D. R., \& Horsey, C. S. (1997). From First Grade Forward: Early Foudations of High School Dropout. Sociology of Education, 70, 87-107. https://doi.org/10.2307/2673158

Bandura, A. (1976). Social Learning Theory. Prentice Hall.

Bandura, A., Freeman, W. H., \& Lightsey, R. (1997). Self-Efficacy: The Exercise of Control. Journal of Cognitive Psychotherapy, 13, 158-166. https://doi.org/10.1891/0889-8391.13.2.158

Bretherton, I., \& Mnholland, K. A. (2008). Internal Working Models in Attachment Relationships: Elaborating a Central Construct in Attachment Theory. In J. Cassidy, \& P. R. Shaver (Eds.), Handbook of Attachment: Theory, Research, and Clinical Applications (pp. 102-127). Guilford Press.

Brody, G. H., Yu, T., Beach, S. R. H., Kogan, S. M., Windle, M., \& Philibert, R. A. (2014). Harsh Parenting and Adolescent Health: A Longitudinal Analysis with Genetic Moderation. Health Psychology, 33, 401-409. https://doi.org/10.1037/a0032686

Brown, K. W., \& Ryan, R. M. (2003). The Benefits of Being Present: Mindfulness and Its 
Role in Psychological Well-Being. Journal of Personality and Social Psychology, 84, 822848. https://doi.org/10.1037/0022-3514.84.4.822

Callahan, K. L., Scaramella, L. V., Laird, R. D., \& Sohr-Preston, S. L. (2011). Neighborhood Disadvantage as a Moderator of the Association between Harsh Parenting and Toddler-Aged Children's Internalizing and Externalizing Problems. Journal of Family Psychology, 25, 68-76. https://doi.org/10.1037/a0022448

Caprara, G. V., Giunta, L. D., Eisenberg, N., Gerbino, M., Pastorelli, C., \& Tramontano, C. (2008). Assessing Regulatory Emotional Self-Efficacy in Three Countries. Psychological Assessment, 20, 227-237. https://doi.org/10.1037/1040-3590.20.3.227

Chen, S. Y., Gui, H., Zhou, R. L., \& Jia, Y. Y. (2012). Revision of Mindful Attention Awareness Scale (MAAS). Chinese Journal of Clinical Psychology, 20, 148-151. https://doi.org/10.16128/j.cnki.1005-3611.2012.02.024

Clark, L. A., Kochanska, G., \& Ready, R. (2000). Mothers' Personality and Its Interaction with Child Temperament as Predictors of Parenting Behavior. Journal of Personality and Social Psychology, 79, 274-285. https://doi.org/10.1037/0022-3514.79.2.274

Deater-Deckard, K., Sewell, M. D., Petrill, S. A., \& Thompson, L. A. (2010). Maternal Working Memory and Reactive Negativity in Parenting. Psychological Science, 21, 75-79. https://doi.org/10.1177\%2F0956797609354073

Deci, E. L., \& Ryan, R. M. (1985). Intrinsic Motivation and Self-Determination in Human Behavior. Springer. https://doi.org/10.1007/978-1-4899-2271-7

Deci, E. L., \& Ryan, R. M. (2008). Facilitating Optimal Motivation and Psychological Well-Being Across Life's Domains. Canadian Psychology, 49, 14-23. https://doi.org/10.1037/0708-5591.49.1.14

Fang, L. T., Shi, K., \& Zhang, F. H. (2008). Research on Reliability and Validity of Utrecht Work Engagement Scale-Student. Chinese Journal of Clinical Psychology, 16, 618-620.

Gong, S. Y., Han, Y., Wang, L. X., Gao, L., \& Xiong, J. M. (2016). The Relationships among Task Value, Academic Emotions and Online Learning Satisfaction. E-Education Research, 37, 72-77. https://doi.org/10.13811/j.cnki.eer.2016.03.010

Grolnick, W. S., Deci, E. L., \& Ryan, R. M. (1997). Internalization within the Family. In J. E. Grusec, \& L. Kuczynski (Eds.), Parenting and Children's Internalization of Values: $A$ Handbook of Contemporary Theory (pp. 135-161). Wiley.

Hayes, A. F. (2013). Introduction to Mediation, Moderation, and Conditional Process Analysis. Journal of Educational Measurement, 51, 335-337. https://doi.org/10.1111/jedm.12050

Jia, X. J., Cai, L., Lin, L., \& Lin, C. D. (2020). The Relationship between Perceived Teachers' Support and Academic Engagement among High School Students: The Chain Mediating Effect of Academic Self-efficacy and Achievement Goal Orientation. Psychological Development and Education, 36, 700-707. https://doi.org/10.16187/j.cnki.issn1001-4918.2020.06.08

Kochanska, G., Murray, K. T., \& Harlan, E. T. (2000). Effortful Control in Early Childhood: Continuity and Change, Antecedents, and Implications for Social Development. Developmental Psychology, 36, 220-232. https://doi.org/10.1037/0012-1649.36.2.220

Laborde, S., Brüll, A., Weber, J., \& Anders, L. S. (2011). Trait Emotional Intelligence in sports: A Protective Role against Stress through Heart Rate Variability? Personality and Individual Differences, 51, 23-27. https://doi.org/10.1016/j.paid.2011.03.003

Lewis, A. D., Huebner, E. S., Reschly, A. L., \& Valois, R. F. (2009). The Incremental Validity of Positive Emotions in Predicting School Functioning. Journal of Psycho Educational Assessment, 27, 397-408. https://doi.org/10.1177\%2F0734282908330571 
Li, D. P., Zhang, W., Li, X., Li, N. N., \& Ye, B. J. (2012). Gratitude and Suicidal Ideation and Suicide Attempts among Chinese Adolescents: Direct, Mediated, and Moderated Effects. Journal of Adolescence, 35, 55-66. https://doi.org/10.1016/j.adolescence.2011.06.005

Li, J. E. (2012). The Relationship among Parenting Style, Student Burnout and Learning Engagement. Unpublished Master's Dissertation, Shandong Normal University.

Li, Y. Z. (2018). The Impact of Parental Rearing Style on Learning Engagement among Senior High School Students: A Serial Mediation Effect Model. Psychological Development and Education, 34, 576-585.

https://doi.org/10.16187/j.cnki.issn1001-4918.2018.05.08

Liu, M. T., \& Wei, H. (2020). The Dark Side of White Lies: Parenting by Lying in Childhood and Adolescent Anxiety, the Mediation of Parent-Child Attachment and Gender Difference. Children and Youth Services Review, 119, Article ID: 105635.

https://doi.org/10.1016/j.childyouth.2020.105635

Martins, A., Ramalho, N., \& Morin, E. (2010). A Comprehensive Meta-Analysis of the Relationship between Emotional Intelligence and Health. Personality and Individual Differences, 49, 554-564. https://doi.org/10.1016/j.paid.2010.05.029

Podsakoff, P. M., MacKenzie, S. B., Lee, J.-Y., \& Podsakoff, N. P. (2003). Common Method Biases in Behavioral Research: A Critical Review of the Literature and Recommended Remedies. Journal of Applied Psychology, 88, 879-903. https://doi.org/10.1037/0021-9010.88.5.879

Ryan, R. M., \& Deci, E. L. (2017). Self-Determination Theory: Basic Psychological Needs in Motivation, Development, and Wellness. Guilford Press. https://doi.org/10.1521/978.14625/28806

Ryan, R. M., Legate, N., Niemiec, C. P., \& Deci, E. L. (2012). Beyond Illusions and Defense: Exploring the Possibilities and Limits of Human Autonomy and Responsibility through Self-Determination Theory. In P. R. Shaver, \& M. Mikulincer (Eds.), Meaning, Morality, and Choice: The Social Psychology of Existential Concerns (pp. 215-233). American Psychological Association. https://doi.org/10.1037/13748-012

Schaufeli, W. B., Salanova, M., González-Romá, V., \& Bakker, A. B. (2002). The Measurement of Engagement and Burnout: A Two Sample Confirmatory Factor Analytic Approach. Journal of Happiness Studies, 3, 71-92.

https://doi.org/10.1023/A:1015630930326

Siff, J. (2014). Thoughts Are Not the Enemy: An Innovative Approach to Meditation Practice. Shambhala.

Sirois, F., \& Pychyl, T. (2013). Procrastination and the Priority of Short-Term Mood Regulation: Consequences for Future Self. Social \& Personality Psychology Compass, 7, 115-127. https://doi.org/10.1111/spc3.12011

Wang, M. T., Degol, J. L., \& Henry, D. A. (2019). An Integrative Development-in-Sociocultural-Context Model for Children's Engagement in Learning. American Psychologist, 74, 1086-1102. https://doi.org/10.1037/amp0000522

Wang, M. Z., Deng, X. L., \& Du, X. X. (2017). Harsh Parenting and Academic Achievement in Chinese Adolescents: Potential Mediating Roles of Effortful Control and Classroom Engagement. Journal of School Psychology, 67, 16-30.

https://doi.org/10.1016/j.jsp.2017.09.002

Wang, M. Z., Du, X. X., \& Zhou, Z. K. (2016). Harsh Parenting: Meaning, Influential Factors and Mechanisms. Advances in Psychological Science, 24, 379-391.

https://doi.org/10.3724/SP.J.1042.2016.00379

Wang, M. Z., Wang, J., Wang, B. Y., Qu, X. Q., \& Xin, F. K. (2020). Harsh Parenting and 
Adolescents' Academic Achievement: A Moderated Mediation Model. Psychological Development and Education, 36, 67-76.

https://doi.org/10.16187/j.cnki.issn1001-4918.2020.01.08

Wang, Y. J., Dou, K., \& Liu, Y. (2013). Revision of the Scale of Regulatory Emotional Self-Efficacy. Journal of Guangzhou University (Social Science Edition), 12, 45-50.

Wei, H., \& Liu, M. T. (2021). Dramaturgical Perspective Mediates the Association between Parenting by Lying in Childhood and Adolescent Depression and the Protective Role of Parent-Child Attachment. Child Abuse \& Neglect, 114, Article ID: 104985. https://doi.org/10.1016/j.chiabu.2021.104985

Wei, J., Liu, R., He, Y., Tang, M., Di, M., \& Zhuang, H. (2014). Mediating Role of Learning Persistence and Engagement in Relations among Self-Efficacy, Intrinsic Value and Academic Achievement. Studies of Psychology and Behavior, 12, 326-332.

Wen, C., Zhang, W., Li, D. P., Yu, C. F., \& Dai, W. Z. (2010). Relationship between Junior Students' Gratitude and Academic Achievement: With Academic Engagement as the Mediator. Psychological Development and Education, 26, 598-605.

Xiong, J. M., Gong, S. Y., \& Frenzel, A. C. (2011). The Relationship between Mathmatics Academic Emotion, Learning Strategies and Mathematics Achievement of Senior High School Students. Educational Research and Experiment, 6, 89-92.

Zhang, N. (2020). The Relationship between Rough Parenting and Learning Engagement: Intentional Self-Regulation and Perceived Teacher Support. Unpublished Master's Dissertation, Guangxi University for Nationalities. https://doi.org/10.27035/d.cnki.ggxmc.2020.000312

Zhou, P. S., Wei, Y. M., \& Yang, K. (2014). A Study on the Relationship between Middle School Students' Educational Style, Learning Engagement and Academic Achievement. Journal of Teaching and Management, 9, 88-90. 